\title{
Transport properties of confined hydrocarbons in slit pores
}

\author{
DR. VASILY V. PISAREV ${ }^{1}$ AND ANDREY G. \\ KALINICHEV ${ }^{1,2}$ \\ ${ }^{1}$ National Research University Higher School of Economics \\ Presenting Author: vpisarev@hse.ru
}

${ }^{2}$ IMT Atlantique

The transport properties of liquid hydrocarbons in slit pores between layers of clay minerals are studied using nonequilibrium molecular dynamics methods. Hydrocarbons and walls are simulated with the OPLS-AA-1.14*CM1A [1] and ClayFF [2] force fields, respectively, and pore widths from 2 to 7 $\mathrm{nm}$ are considered.

Non-equilibrium molecular dynamics simulations are performed for Couette and Poiseuille flows. For Couette flow, the MüllerPlathe approach [3] is used. To simulate a constant pressure gradient for Poiseuille flow, equal forces are applied to each molecule of the fluid. From the velocity profiles for flows, shear viscosity of the fluid and fluid-wall slip length are calculated. The viscosity-density dependencies for various pore widths are constructed by taking the Voronoi tessellation of the system and considering the total number of Voronoi cells centered on molecules of fluid as the fluid volume.

The simulations show that the viscosity-density dependence of the fluid is practically independent of the distance between pore walls, even for the smallest widths $(2-3 \mathrm{~nm})$ The slip length is of the order of the intermolecular distance, which can be significant for continuum simulations of fluids in nanoscale pores.

The study was implemented in the framework of the Basic Research Program at the National Research University Higher School of Economics (HSE University) in 2021.

[1] Dodda, Vilseck, Tirado-Rives \& Jorgensen (2017), J. Phys. Chem. B 121, 3864-3870

[2] Cygan, Liang \& Kalinichev (2004) J. Phys. Chem. B 108, $1255-1266$

[3] Müller-Plathe (1999), Phys. Rev. E 59, 4894 